\title{
The Design of 150 W Regulated Power Supply Based on Principle of the Electronic Transformer
}

\author{
Wu Shide ${ }^{1}$, Yang Jinfeng ${ }^{2}$, Ma Zeyu ${ }^{1}$ \\ 1. College of Mechanical and Transportation Engineering; \\ 2. College of Geophysics and Information Engineering \\ China University of Petroleum, Beijing, China \\ E-mail: wusde@cup.edu.cn/yangjinfeng_cup@163.com/martinzyly@126.com
}

\begin{abstract}
In this paper, the operation principle of the electronic transformer and the factor influenced the switching frequency of electronic transformer are introduced in detail. Meanwhile a regulated power supply which is reformed by electronic transformer is proposed. Using voltage and current feedback, it realizes the constant output voltage and the over-current protection. The feedback theory is given in detail. The $150 \mathrm{~W}$ regulated power supply with the advantages of simple structure, light weight and low cost is developed.
\end{abstract}

Keywords-electronic transformer; magnetic saturation; switching frequency; regulated power supply.

\section{INTRODUCTION}

Currently, the switching power supply gradually replaces the traditional linear power supply or the SCR power supply and the power supply using in varieties of electronic equipments has been almost dominated by it, as in [1]. In the range of small and medium power, the forward switching power supply and the flyback switching power supply have been commonly adopted, due to the advantages of simple drive circuit, a small number of components, and low cost, as in [2]. The drawbacks are that the operating point of magnetic core for the transformer is in I quadrant of the magnetization curve plane, the utilization ratio of the magnetic core is low and the current and voltage peak value which the switching devices withstand is large.

Widely using in quartz spotlights, the electronic transformer which has the advantages of light weight, simple circuit and low cost is a push-pull switching power supply in essence, as in [3]. It also has the more advantages, comparing with the forward switching power supply and the flyback switching power supply: (1) the simple driver circuit, using the mutual coupling inductance coil winding in the magnetic ring to produce square waves which drive switch devices; (2) the bi-directional excitation transformer which means that the utilization ratio of magnetic core is higher and the size of the magnetic core is smaller; (3) no control chip. As the drive of adapter for the quartz spotlights, the output of the electronic transformers is AC square wave. Therefore, it is an open-loop control and high-frequency electronic transformer in essence. Based on electronic transformers, the regulated power supply with voltage and current feedback control for small and medium power has a distinct advantage and absolutely has a good practicality in the market, comparing with the forward power supply and the flyback power supply.

\section{OPERATION STATE ANALYSIS FOR THE ELECTRONIC TRANSFORMER}

The schematic diagram of the electronic transformer is showed in Fig. 1. Winding on the same magnetic ring T1, $\mathrm{T} 1 \mathrm{~b}$ and $\mathrm{T} 1 \mathrm{c}$ generate a square wave and trigger the conduction of Q1 and Q2. After circuit is electrified and the voltage of $\mathrm{C} 1$ charges to $32 \mathrm{~V}, \mathrm{DB} 3$ is on, as in [4] and Q2 turns on. When Q2 is on, DB3 has no effect because the magnetic ring can generate drive square waves which let Q1 and Q2 conduct in turn. A cycle can be divided into six operation states. The main waveform is shown in Fig. 2. The transition process is amplified in order to facilitate the analysis.

\section{A. Operation state $1\left(t_{0}, t_{1}\right)$}

At time $t_{0}$, the transistor Q2 is in the saturated region and $\mathrm{Q} 1$ is in the cutoff region. The direction of current flow is BQ2-C-C3-T2-T1a-B. The excitation current of the winding T1a grows linearly according to (1). If the capacitance value of $\mathrm{C} 2$ and $\mathrm{C} 3$ is large enough, the voltage ripple of $\mathrm{C} 2$ and $\mathrm{C} 3$ is small. The equation of $U_{B G}, U_{F B}$ and $i_{T l a}$ can be expressed as following:

$$
\begin{gathered}
U_{B G}=L_{T 1 a} \frac{d i_{T 1 a l}}{d t}+L_{T 2} \frac{d i_{T 2 l}}{d t} \approx L_{T 2} \frac{d i_{T 2 l}}{d t}=\frac{U_{A C}}{2} \approx-150(\mathrm{~V}) \\
U_{F B}=L_{T 1 a} \frac{d i_{T 1 a l}}{d t}=\frac{U_{b e}}{n} \approx \frac{1}{3} \times 0.7(\mathrm{~V}) \\
i_{T 1 a}=i_{T 1 a l}+i_{T 1 a f}
\end{gathered}
$$

Where, $i_{T l a}$ is the total current of T1a, $i_{\text {Tlal }}$ is the excitation current of T1a, $i_{\text {Tlaf }}$ is the driving current of the load and $i_{T 2 a l}$ is the excitation current of $\mathrm{T} 2$.

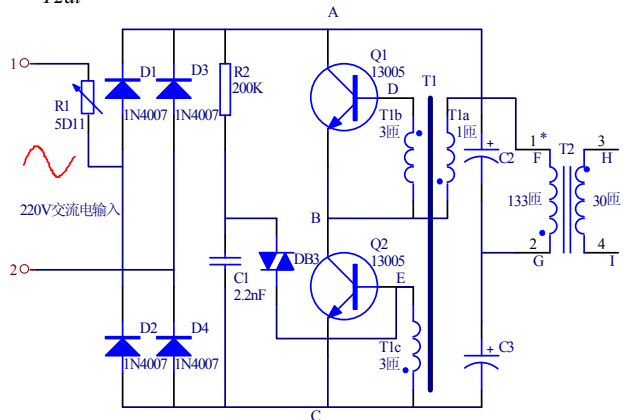

Figure 1. Schematic diagram of the electronic transformer 


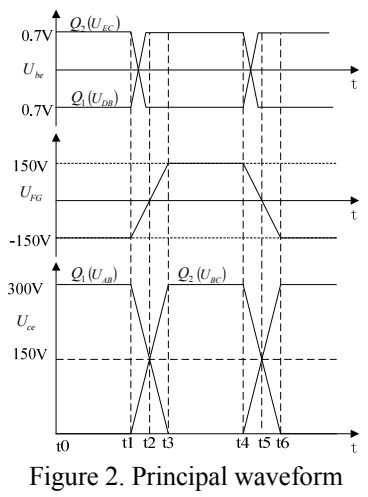

B. Operation state $2\left(t_{1}, t_{2}\right)$

At time $t_{1}$, due to effect of the excitation current, the magnetic core $\mathrm{T} 1$ tends to magnetic saturation. As the rate of change for the magnetic flux decreases sharply, tending to magnetic saturation stage according to the $\mathrm{B}-\mathrm{H}$ curve, the excitation current increases sharply and the driving current for Q2 declines sharply. Meanwhile, the magnetic core is close to magnetic saturation, which causes the inductive voltage of T1c being small not to sustain conduction of Q2. Therefore, Q2 turns off. In the interval of $t_{1}-t_{2}$, when the voltage $U_{b e}$ is zero, the magnetic ring begins to desaturate and when the voltage of T1 a decreases sharply, T1b and T1c are induced negative electromotive force according to the Lenz's law. Before reaching the time $t_{2}$, the magnetic ring has exited in saturation region and entered into the linear region. In this interval, Q1 and Q2 are in amplified area. At time $t_{2}, i_{A B}=i_{B C}, i_{T l a}=0, \mathrm{U}_{\mathrm{AB}}=\mathrm{U}_{\mathrm{BC}}=\frac{U_{A C}}{2} \approx-150(\mathrm{~V})$.

\section{Operation state $3\left(t_{2}, t_{3}\right)$}

From the time $t_{2}$, the excitation current $i_{\text {Tlal }}$ increases linearly in reverse direction. Q1 and Q2 are still in amplified area. In this interval, $I_{A B}$ is greater than $I_{B C}$ and $i_{T I a}$ increases reversely. At time $t_{3}, \mathrm{Q} 2$ changes to the cutoff region from the amplified area and Q1 changes to the saturated region from the amplified area.

\section{Operation state $4\left(t_{3}, t_{4}\right)$}

Q1 is in the saturated region and Q2 is in the cutoff region in this interval. The direction of current flow is A-Q1-T1a-T2-C2-A. The excitation of T1a increases linearly in reverse direction. Then the equations can been gotten as following:

$$
\begin{gathered}
U_{F G}=L_{T 1 a} \frac{d i_{T 1 a l}}{d t}+L_{T 2} \frac{d i_{T 2 l}}{d t} \approx L_{T 2} \frac{d i_{T 2 l}}{d t}=\frac{U_{A C}}{2} \approx 150(\mathrm{~V}) \\
U_{F B}=L_{T 1 a} \frac{d i_{T 1 a l}}{d t}=\frac{U_{b e}}{n}=-\frac{1}{3} \times 0.7(\mathrm{~V})
\end{gathered}
$$

\section{E. Operation state $5\left(t_{4}, t_{5}\right)$}

The principle of this operation state is similar to the operation state 2.

\section{F. Operation state $6\left(t_{5}, t_{6}\right)$}

The principle of this operation state is same as the operation state 3 . When this operation state finishes, it enters into the next cycle.

\section{FACTORS OF AFFECTING SWITCHING FREQUENCY}

The relationship of the excitation current, the magnetic flux, and the induced voltage can be obtained by the Faraday law of electromagnetic induction, as shown in (3). The volt-second value can be calculated by (3), as shown in (4). When the magnetic core has been to magnetic saturation, the variable quantity of the magnetic flux is a fixed value, as shown in (5). Equation (6) is an expression of the switching frequency.

$$
\begin{gathered}
L \frac{d i_{T 1 a l}}{d t}=N \frac{d \Phi}{d t}=U_{F B} \\
N \Delta \Phi=U_{F B} \Delta t_{r} \\
\Delta \Phi=2 B_{S} A \\
\Delta t_{r}+\Delta t_{e}=\frac{T}{2}=\frac{1}{2 \mathrm{f}}
\end{gathered}
$$

Where, $B_{S}$ is the saturated magnetic induction density of the magnetic ring, $A$ is the cross-sectional area of the magnetic ring, $\Delta t_{r}$ is the time from cutoff region to saturated region for transistor and $\Delta t_{e}$ is the time from saturated region to cutoff region for transistor.

The time for $\Delta t_{r}$ and $\Delta t_{e}$ is related to the load current. The greater the load current is, the larger the base drive current of the transistor is. As the base drive current increases, the saturation level of the transistor becomes deeper and the desaturation time of the transistor gets longer which means the time for $\Delta t_{r}$ and $\Delta t_{e}$ becomes long and the switching frequency becomes small. The factors of affecting the switching frequency are the turn number of the magnetic ring, the base resistance, the input voltage, the load size and the ambient temperature. To be specific, the switching frequency changes with these factors as below:

$A$ 、 As the turn number (T1c or T1b) of the magnetic ring decreases, the input induced voltage $U_{F B}$ of the magnetic ring increases because of the constancy of the transistor's base voltage $U_{b e}$. Therefore, $\Delta t_{r}$ gets small inevitably and the switching frequency becomes high, as shown in (4) and (6). $B$, When the base resistance is increased, the secondary induced voltage $U_{E C}$ of the magnetic ring increases and the primary induction voltage $U_{F B}$ increases as well. Therefore, $\Delta t_{r}$ decreases and the switching frequency increases.

$C$. When the input voltage decreases, the primary current of the transformer decreases because the output current decreases. Due to the constancy of the excitation current of the magnetic ring, the drive current inevitably reduced, as shown in (2). Therefore, the switching frequency increases.

$D$. When the load gets heavy, the primary current of the transformer increases. Due to the constancy of the excitation current of the magnetic ring, the driving current inevitably increases. Therefore, the switching frequency decreases.

$E$ 、 When the ambient temperature is raised, the base voltage $U_{b e}$ of the transistor reduces, as in [5] and the primary induction voltage $U_{F B}$ gets small. As the time $\Delta t_{r}$ increases, the switching frequency decreases.

The turn number of the magnetic ring should be guaranteed that the volt-seconds value of the main transformer core (T2) meets the design requirements in the rated operation load. Decreasing the time $\Delta t_{r}$ can reduce the 
volt-second value of the main transformer by reducing secondary turn number, changing the turn ratio of the magnetic ring, reducing the core area or adding resistance in base of the transistor.

\section{FeEdBACK LoOP Design}

Changing the transformer $\mathrm{T} 2$ and increasing the voltage comparator etc., electronic transformer in Fig. 1 can be reformed into a regulated power supply in Fig. 3. The feedback loop design can be realized by the effect of the magnetic ring. When the output voltage or current is higher than the set value, Q3 turns on and the magnetic ring T1d is shorted. Because all the output power of the magnetic consumes in winding $\mathrm{T} 1 \mathrm{~d}$, the current and the induced voltage of $\mathrm{T} 1 \mathrm{c}$ and $\mathrm{T} 1 \mathrm{~b}$ is so tiny that the triode turns off. In short, controlling magnetic ring (T1d) by Q3, the feedback control of the voltage and current can be realized.

The reference voltage $2.5 \mathrm{~V}$ for LM393's pin 3 is supplied by TL431. When the output voltage is greater than $30 \mathrm{~V}$, the voltage of pin 2 is greater than the voltage of pin 3 and then the voltage of pin 1 becomes zero. When the dotted terminal of T1d induces the negative voltage and Q3 conducts, the magnetic ring T1d is shorted and the triode Q2 turns off. Therefore, the output voltage decreases until the voltage of pin 2 is equal to the voltage of pin 3, which means the output voltage value being constant $30 \mathrm{~V}$. The principle of current feedback is same as the voltage feedback.

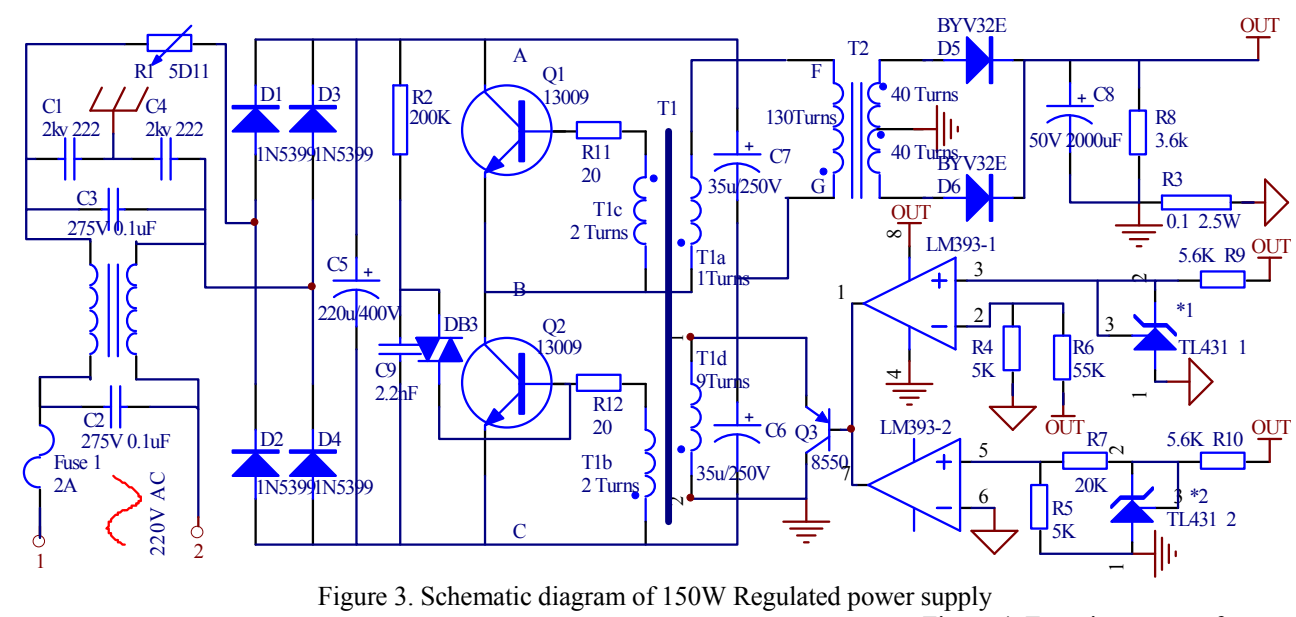

\section{EXPERIMENT RESULTS}

All the specific parameters of the regulated power supply have been given in Fig. 3. The output voltage ripple of the power supply is approximately $1 \%$ on condition in rated power. The material of the main transformer core is the PC40 and the EI33/29/13 core is used. The material of the magnetic ring is the $\mathrm{Mn}-\mathrm{Zn}$ ferrite.

When the AC line voltage is $220 \mathrm{~V}$ and the power is $150 \mathrm{~W}$, the main waveform is measured as shown in Fig. $4 \mathrm{a}$ $\sim 4 \mathrm{~d}$. Because the voltage waveform $U_{c e}$ and $U_{b e}$ of Q1 and $\mathrm{Q} 2$ are symmetrical, the figure only gives a group of Q2. As

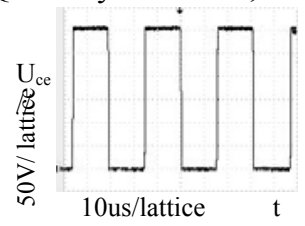

(a) The tube voltage waveform of Q2

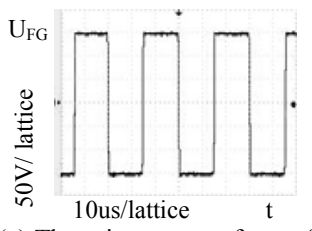

(c) The primary waveform of the main transformer

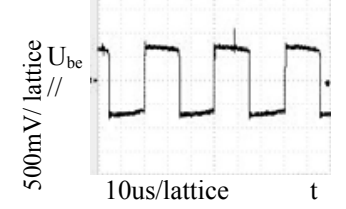

(b) The drive waveform of Q2

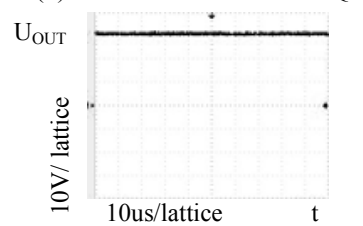

(d) The output voltage waveform can be seen from Fig. $4 \mathrm{a} \sim 4 \mathrm{c}$, the waveforms are the same as the theoretical analysis of the waveform in Fig. 2. Seen from Fig. $4 d$, the output voltage is $30 \mathrm{~V}$.

\section{CONCLUSION}

The principle of the high-frequency electronic transformer is analyzed in detail. Based on the principle of electronic transformers, the designing and test for the $150 \mathrm{~W}$ regulated power supply have finished. The theoretical analysis and the experimental results show that the designing of the new switching power supply have following characteristics: (1) it has the feedback control of the voltage and the current; (2) the excitation of the transformer is bi-directional, the utilization ratio of the core is high and the size of the core is small; (3) the main circuit of the power supply is half-bridge structure, but a simpler drive circuit; (4) the method for the drive circuit and the feedback control provides a new thought for the improvements of the switching power supply.

\section{REFERENCE}

[1] Zhang Enhuai. The Development and the Summarizing of Switching Power Supply. Power Electronics, 1996 (01).

[2] Yang $\mathrm{Xu}$, Pei Yunqing, Wang Zhaoan. Switching Power Supply Technology. Mechanieal Industrial Press, Beijing. pp. 112-113, 2005. 
$U_{F B}=L_{T 1 a} \frac{d i}{c}$

[3] Wang Han, YAN Caiyan, YOU Longxiang. The Development of a New Electronic Transformer. Journal of Xiamen University (Natural Science Edition), pp. 303-305, 2005.

[4] Chen Yutian, CHEN Aijun, CHEN Aiquan etc. 100 Examples for Making and Maintenance of the Efficient Energy-saving Electron
Fluorescent Lamp. Posts and Telecommunications Press, Beijing, pp. 325-326, 1999.

[5] Chen Chuanchu. Principle and Making of Energy-saving Electron Lamp and Electronic Ballast. Posts and Telecommunications Press, Beijing, pp. 44-45, 2004. 